\title{
On the Causality and Stability of the Relativistic Diffusion Equation
}

\author{
Peter Kostädt and Mario Liut \\ Institut für Theoretische Physik, Universität Hannover, \\ 30167 Hannover, Germany
}

(Phys. Rev. D 62, 023003 (2000))

\begin{abstract}
This paper examines the mathematical properties of the relativistic diffusion equation. The peculiar solution which Hiscock and Lindblom identified as an instability is shown to emerge from an ill-posed initial value problem. These do not meet the mathematical conditions required for realistic physical problems and can not serve as an argument against the relativistic hydrodynamics of Landau and Lifshitz.
\end{abstract}

47.75.+f, 02.30.Jr, 03.30.+p, 05.70.Ln

\section{INTRODUCTION}

The relativistic generalization of the hydrodynamic theory, as developed by Landau and Lifshitz [1], leads to differential equations of the parabolic type. Consider for instance the diffusion equation for the viscous shear flow. In a frame in which the background equilibrium state is at rest ("comoving frame"), it is

$$
\left[c^{-2}(e+p) \partial_{t}-\eta \partial_{x}^{2}\right] \delta u^{i}(x, t)=0,
$$

where $\delta u^{i}$ with $i=y, z$ is the perturbation in the transverse velocity. Equation (11) is the archetype of a parabolic equation. In non-relativistic physics, it provides an excellent description of a wide range of physical phenomena, as countless experiments have shown. Within the relativistic framework, however, it seems to fail, as two deficiencies become evident: The first, acausality, refers to the fact that Eq. (1i) allows for propagation of signals with arbitrarily large velocities. (This should be, and indeed has already been, a worry in the Galilean hydrodynamics, since one has very definite ideas of the velocities of the constituent microscopic particles, which represent an upper limit of the signal velocity in a dilute system of hard-core interaction.) The second defect is an instability found by Hiscock and Lindblom [2], who showed that Eq. (11) develops a solution that grows exponentially with time in any non-comoving Lorentz frames. It is noteworthy that the growth time scale was found to be microscopically short.

To overcome these deficiencies, extended fluid theories were put forward which start from the hydrodynamic theory but include additional dynamic variables; see e.g. [3]. The resultant larger set of phenomenological coefficients can be chosen such that all the equations are hyperbolic, ensuring causality and stability. The price for this is twofold: A rather more complicated theory and the difficulty of finding a universally accepted set of additional variables (except perhaps in dilute systems). In fact, recently it has been shown by Geroch [- 4 and Lindblom [5] that the complicated dynamical structure which ensures causality is unobservable. The evolution of any physical fluid state according to any causal theory results in energy-momentum tensors and particle currents that are experimentally indistinguishable from the respective hydrodynamic expressions.

In this paper, we take a step back and again focus on the simpler and more universal parabolic equation (11). While acausality is an expected feature of parabolic differential equations, already discussed in the non-relativistic context [6 8], instability is not. Being absent in a comoving frame, one is astonished at its appearance in non-comoving frames. Our main purpose thus is to examine the origin and physical relevance of the instability.

The paper is organized as follows: In Sec. II some general mathematical aspects of partial differential equations are reviewed. We especially recall the intuitive meaning of the characteristics. Section III is devoted to the onedimensional diffusion equation in a comoving frame. We discuss the problem of causality and examine the two types of Cauchy problems that can be formulated with respect to a parabolic equation. In Sec. IV the discussion is generalized to non-comoving Lorentz frames. We especially scrutinize the solution that Hiscock and Lindblom have identified as an instability, and show that it is a result of an ill-posed initial value problem. In Sec. V we briefly examine the general case, in which the spatial dimension of the diffusion equation is greater than one.

\section{PRELIMINARIES}

Let us first review some general aspects of partial differential equations that can be found in standard textbooks on mathematical physics (eg. [9]). Consider a linear partial differential equation of second order for the unknown function $\vartheta(x, t)$. It can be written, most generally, in the form

$$
\left[A \partial_{x}^{2}+2 B \partial_{x} \partial_{t}+C \partial_{t}^{2}\right] \vartheta+F\left(x, t, \vartheta, \partial_{x} \vartheta, \partial_{t} \vartheta\right)=0
$$

where $A, B$ and $C$ are given functions of the two independent variables $x$ and $t$. Depending on the value of $D \equiv B^{2}-A C$ at a given point, Eq. (2) is referred to be of the elliptic $(D<0)$, parabolic $(D=0)$, or hyperbolic 
$(D>0)$ type in this point. In the following we shall restrict ourselves to the cases in which $A, B$ and $C$ are constants. The type of Eq. (2) then remains unchanged throughout the entire region.

The equation

$$
A\left(\partial_{x} \varphi\right)^{2}+2 B\left(\partial_{x} \varphi\right)\left(\partial_{t} \varphi\right)+C\left(\partial_{t} \varphi\right)^{2}=0
$$

is called the equation of characteristics of the partial differential equation (2). Correspondingly, the family of curves,

$$
\varphi(x, t)=\text { const },
$$

with $\varphi(x, t)$ satisfying Eq. (3), is called the family of characteristics. We collect the following facts:

(i) An equation of the hyperbolic type has two distinct families of real characteristics, an equation of the parabolic type has only one; an elliptic equation does not have real characteristics.

(ii) The equation of characteristics is invariant with respect to arbitrary transformations of the independent variables, $\tilde{x}=\tilde{x}(x, t), \tilde{t}=\tilde{t}(x, t)$. This implies that, if $\varphi(x, t)$ is a solution of Eq. (3), and if $\varphi(x, t)$ transforms into $\tilde{\varphi}(\tilde{x}, \tilde{t})$, then $\tilde{\varphi}(\tilde{x}, \tilde{t})$ is a solution of the equation of characteristics accompanying the transformed differential equation.

(iii) The outer real characteristics that pass through a given point $\left(x_{0}, t_{0}\right)$ bound the domain of influence $\Omega_{0}$ of this point. If we consider the variable $t$ as the time and think of the solution $\vartheta(x, t)$ as a quantity that varies in $x$-space with time $t$, then this means that for $t>t_{0}$ the solution in the region outside $\Omega_{0}$ is not influenced by the initial data given at $\left(x_{0}, t_{0}\right)$. As an example, consider the telegraph equation

$$
\partial_{t} \vartheta-\alpha\left(\partial_{x}^{2}-\frac{1}{v^{2}} \partial_{t}^{2}\right) \vartheta=0
$$

with given constants $\alpha, v>0$. Since $D=\alpha^{2} / v^{2}>0$, it belongs to the hyperbolic type. Its equation of characteristics is $\left(\partial_{x} \varphi\right)^{2}-\left(\partial_{t} \varphi\right)^{2} / v^{2}=0$, which gives two families of characteristics,

$$
\varphi(x, t)=x \pm v t=\text { const. }
$$

Taking some point $\left(x_{0}, 0\right)$, the respective domain of influence is thus bounded by $x \pm v t=x_{0}$, implying that the effects of the initial data propagate at finite velocity $v$.

For equation (2), the most general Cauchy problem is formulated in the following way: Let $S$ be some smooth curve given in the space of the variables $x, t$. With each point $(x, t) \in S$ there is associated some direction $n$ not tangent to $S$. The problem now consists of finding, in some neighborhood of $S$ (either on one or both sides of the curve), a solution $\vartheta(x, t)$ which satisfies the prescribed Cauchy conditions

$$
\left.\vartheta\right|_{S}=\Theta_{0}(x, t),\left.\quad \frac{\partial \vartheta}{\partial n}\right|_{S}=\Theta_{1}(x, t) .
$$

It is important to note that the domain in which the unknown solution has to be determined is not specified beforehand. So generally, the initial manifold $S$ lies within the domain of definition of the solution.

A problem is said to be well-posed if it has the following properties: The solution (i) exists, (ii) is uniquely determined, and (iii) depends continuously on the assigned data. The last requirement is imposed in connection with the fact that the initial data of physical problems are determined experimentally and so small errors occur. It is thus necessary to be sure that the solution does not depend essentially on the measurement errors of these data.

\section{THE DIFFUSION EQUATION IN A COMOVING FRAME}

A well-known example of Eq. (2) is given by the parabolic diffusion equation

$$
\partial_{t} \vartheta-\alpha \partial_{x}^{2} \vartheta=0
$$

where $\alpha>0$. With $A=-\alpha$ and $B=C=0$ its equation of characteristics (3) takes the form $\left(\partial_{x} \varphi\right)^{2}=0$. Hence, the characteristics are given by the one-parameter family of lines

$$
\varphi(x, t)=t=\text { const }
$$

It is obvious from this that the diffusion equation allows for the propagation of disturbances with infinite velocity. Indeed, the initial value of $\vartheta$ at the point $\left(x_{0}, t_{0}\right)$ has influence on the solution $\vartheta(x, t)$ in the whole semi-infinite region $t \geq t_{0}$.

This fact, however, does not in practice cause any complications: The superluminal propagation speeds are associated only with variations on microscopically small time and length scales (and with amplitudes of the order of thermodynamic fluctuations). On these scales a macroscopic description loses validity, and the diffusion equation and its solutions break down accordingly. The acausal consequences are therefore precluded by restricting the solutions of Eq. (8) to the hydrodynamic range of validity,

$$
\left|\frac{\partial_{x} \vartheta}{\vartheta-\vartheta_{0}}\right| \ll \frac{1}{\xi}, \quad\left|\frac{\partial_{t} \vartheta}{\vartheta-\vartheta_{0}}\right| \ll \frac{1}{\tau},
$$

with $\vartheta_{0}$ denoting the constant part of $\vartheta$; and $\xi, \tau$ the characteristic distance and time between collisions of particles, or elementary excitations. For gases this has been first demonstrated by Weymann [6].

As an example, consider the solution of Eq. (8) that satisfies the initial condition $\left.\vartheta\right|_{t=0}=\vartheta_{0}+(A / d) e^{-x^{2} / 2 d^{2}}$. It is

$$
\vartheta(x, t)=\vartheta_{0}+\left(A / \sqrt{d^{2}+2 \alpha t}\right) e^{-x^{2} / 2\left(d^{2}+2 \alpha t\right)},
$$


which represents a Gaussian distribution with width $\sqrt{d^{2}+2 \alpha t}$. As can be seen from the first of Eq. (10), this solution is of physical significance only in the interval

$$
|x| \ll d^{2} / \xi+(2 \alpha / \xi) t
$$

The maximum speed at which measurable information is transmitted is thus of the order of $v_{\max } \sim \alpha / \xi$, which is far less than the speed of light for typical values of the kinetic coefficient $\alpha$ and the microscopic length $\xi$. (Taking $\alpha$ as the heat conductivity, one has for iron at room temperature: $\alpha \approx 10^{-5} \mathrm{~m}^{2} / \mathrm{s}$ and $\xi \approx 10^{-8} \mathrm{~m}$. So $v_{\max } \approx 10^{3} \mathrm{~m} / \mathrm{s}$, which is of the order of the speed of sound.)

The most common initial-value problem for Eq. (8) is to find a solution $\vartheta(x, t)$ for which the Cauchy conditions (7) are prescribed at the initial manifold $t=0$. However, since the line $t=0$ is a characteristic here, the Cauchy data cannot be prescribed independently, but must satisfy a compatibility condition [9]. For instance, if the Cauchy conditions are given by $\left.\vartheta\right|_{t=0}=\Theta_{0}(x)$ and $\left.\left(\partial_{t} \vartheta\right)\right|_{t=0}=\Theta_{1}(x)$, we get from Eq. (8), for $t=0$, $\Theta_{1}(x)=\alpha \partial_{x}^{2} \Theta_{0}(x)$. The characteristic Cauchy problem for the diffusion equation is therefore posed in the following way: In the region $t>0$ find a solution $\vartheta(x, t)$ satisfying the initial condition

$$
\left.\vartheta\right|_{t=0}=\Theta_{0}(x)
$$

As is generally known [9], this problem is well-posed for arbitrary, smooth functions $\Theta_{0}(x)$ that have a welldefined Fourier transform. In the special case of a pure exponential, $\Theta_{0}=A e^{i k x}(k \in \mathbb{R})$, the solution takes the form

$$
\vartheta(x, t)=A e^{i k x+\Gamma t}, \quad \Gamma(k)=-\alpha k^{2},
$$

which is unique and stable for $t>0$.

Quite another situation arises when the Cauchy data are prescribed on a non-characteristic curve, say eg. $x+$ $b t=0$, with some finite $b \in \mathbb{R}$. The diffusion equation then gives two modes, one bound and the other divergent with $|x+b t| \rightarrow \infty$. A well-known example is the so-called "sideways problem": In the half-space $x>0(-\infty<t<$ $\infty)$ we seek a function $\vartheta(x, t)$, which satisfies Eq. (8), and which attains on the non-characteristic line $x=0$ the Cauchy conditions

$$
\left.\vartheta\right|_{x=0}=A e^{i \omega t},\left.\quad\left(\partial_{x} \vartheta\right)\right|_{x=0}=B e^{i \omega t},
$$

with $\omega \in \mathbb{R}$. As can be easily seen, this problem is generally solved by

$$
\begin{gathered}
\vartheta(x, t)=\left(\delta \vartheta_{1} e^{(i+1) \Lambda_{1} x}+\delta \vartheta_{2} e^{(i+1) \Lambda_{2} x}\right) e^{i \omega t}, \\
\Lambda_{1,2}(\omega)= \pm \sqrt{\omega / 2 \alpha}
\end{gathered}
$$

which represents a superposition of two modes

$$
\vartheta_{1,2}(x, t)=\delta \vartheta_{1,2}(\omega) e^{i( \pm x \sqrt{\omega / 2 \alpha}+\omega t)} e^{ \pm x \sqrt{\omega / 2 \alpha}},
$$

with the amplitudes $\delta \vartheta_{1,2}(\omega)$ being determined by the two Cauchy conditions (15). One has

$$
\delta \vartheta_{1,2}(\omega)=\frac{1}{2} A \pm \frac{1}{4} B(i-1) \sqrt{2 \alpha / \omega} .
$$

So, without imposing further restrictions, the general solution (16) explodes exponentially as $x$ increases. Moreover, it shows discontinuous dependence on the initial data [10]: As $\omega \rightarrow \infty$, Eq. (16) is bounded on the initial line $x=0$ but grows like $\exp (x \sqrt{\omega / 2 \alpha})$ for any $x>0$. Consequently, the non-characteristic Cauchy problem (15) is not well-posed.

Recalling the intuitive concept of the characteristics (cf. Sec. III), the reason for this becomes obvious. The initial data given at some point $\left(0, t_{0}\right)$ affect the value of the solution exactly in those points which lie in the domain of influence $\Omega_{0}=\left\{(x, t) \mid x \in \mathbb{R}, t \geq t_{0}\right\}$. Solving Eqs. (8), (15) thus yields the solution for $x>0$ as well as for $x<0$. Confer the two modes of Eq. (18). They describe damped waves which carry the initial data given at $x=0$ to the left and to the right. Physical intuition suggests, however, that $\vartheta_{1}$ and $\vartheta_{2}$ do exist only for $x<0$ and $x>0$, respectively. A superposition in the form of Eq. (16) does not make any physical sense.

Now, the sideways problem will not be well-posed unless the behavior at infinity is prescribed. In fact, the physically realistic assumption that $\vartheta$ be bounded as $x \rightarrow \infty$ 1] leads to a solution that exists, is unique, and depends continuously on the initial data given at $x=0$. The correct formulation of the sideways problem thus is the following: Find the bounded solution of Eq. (\&) in the region $x>0(-\infty<t<\infty)$, satisfying

$$
\left.\vartheta\right|_{x=0}=A e^{i \omega t}, \quad \omega \in \mathbb{R} .
$$

Note that the boundedness condition "replaces" the second Cauchy condition.

Equation (15) is an example of a non-characteristic Cauchy problem with the initial data given on a line that is timelike. For the following, it is important to consider also the case where the Cauchy data are given on a spacelike line. As we shall see, this problem is not well-posed either.

In the half-space $\{(x, t) \mid x \in \mathbb{R}, c t-\beta x>0\}$ we seek the solutions of Eq. (8) satisfying periodic initial data on the non-characteristic spacelike line $c t-\beta x=0(\beta \in$ $\mathbb{R}, 0<\beta<1)$. Here $c$ is the speed of light. With $\gamma \equiv\left(1-\beta^{2}\right)^{-1 / 2}, x^{\mu}=(x, c t), \eta^{\mu \nu}=\operatorname{diag}(1,-1)$, and $n^{\mu}=-\gamma(\beta, 1), e^{\mu}=\gamma(1, \beta)$ respectively denoting the timelike and spacelike unit vector normal and parallel to the initial line $c t-\beta x=n^{\mu} x_{\mu}=0$, the general Ansatz

$$
\vartheta(x, t) \sim e^{i k e^{\mu} x_{\mu}+\Gamma n^{\mu} x_{\mu} / c}, \quad k \in \mathbb{R}, \Gamma \in \mathbb{C}
$$

takes the form

$$
\vartheta(x, t) \sim e^{i k \gamma(x-\beta c t)+\Gamma \gamma(t-\beta x / c)},
$$


with $\left.\vartheta\right|_{c t-\beta x=0} \sim \exp \left(i k \gamma^{-1} x\right)$. Inserting Eq. (22) into the diffusion equation (8) yields

$$
\gamma \alpha \frac{\beta^{2}}{c^{2}} \Gamma^{2}-\left(1+2 i \gamma \alpha \frac{\beta}{c} k\right) \Gamma-\gamma \alpha k^{2}+i \beta c k=0 .
$$

For $k \neq 0$ the two roots $\Gamma_{1,2}(k)$ are complex. The real parts are given by

$$
\Gamma_{\mathrm{R} 1, \mathrm{R} 2}=\frac{1}{2} C \pm \sqrt{\frac{1}{8} C^{2}+\sqrt{\frac{1}{64} C^{4}+\frac{1}{4} C^{3} \gamma^{-3} \alpha k^{2}}},
$$

where $C \equiv\left(\gamma \alpha \beta^{2} / c^{2}\right)^{-1}$. From this one finds the inequalities

$$
\begin{gathered}
\Gamma_{\mathrm{R} 1}+\Gamma_{\mathrm{R} 2}=C>0 \\
\Gamma_{\mathrm{R} 1} \Gamma_{\mathrm{R} 2}=\frac{1}{8} C^{2}-\sqrt{\frac{1}{64} C^{4}+\frac{1}{4} C^{3} \gamma^{-3} \alpha k^{2}} \leq 0,
\end{gathered}
$$

which imply that exactly one mode grows exponentially with time. Now, as $k \rightarrow \infty$, the general solution diverges like $\exp \left(\gamma \sqrt{c^{3} / 2 \alpha \beta}[t-\beta x / c] \sqrt{k}\right)$ for any $t>\beta x / c$, while the initial values are bounded on $t-\beta x / c=0$. Hence small changes in the initial data would cause considerable changes in the solution, indicating that the problem is not well-posed.

\section{THE DIFFUSION EQUATION IN A GENERAL LORENTZ FRAME}

Next we turn our attention to the diffusion equation in an inertial frame $\tilde{K}$, in which the medium moves with constant velocity $v$ in the negative $x$-direction. Employing the Lorentz transformation rules $\partial_{t}=\gamma \partial_{\tilde{t}}-\gamma v \partial_{\tilde{x}}$ and $\partial_{x}=\gamma \partial_{\tilde{x}}-\gamma\left(v / c^{2}\right) \partial_{\tilde{t}}$, we get from Eq. (8) the boosted diffusion equation

$$
\gamma\left(\partial_{\tilde{t}}-v \partial_{\tilde{x}}\right) \vartheta-\alpha \gamma^{2}\left(\partial_{\tilde{x}}^{2}-2 \frac{v}{c^{2}} \partial_{\tilde{x}} \partial_{\tilde{t}}+\frac{v^{2}}{c^{4}} \partial_{\tilde{t}}^{2}\right) \vartheta=0 .
$$

It is straightforward to verify that this equation still belongs to the parabolic type. In a covariant language, it is rewritten as

$$
u^{\mu} \partial_{\mu} \vartheta-\alpha \Delta^{\mu \nu} \partial_{\mu} \partial_{\nu} \vartheta=0,
$$

where $\Delta^{\mu \nu}=\eta^{\mu \nu}+c^{-2} u^{\mu} u^{\nu}, u^{\mu}=\gamma(-v, c), \partial_{\mu}=$ $\left(\partial_{\tilde{x}}, \partial_{c \tilde{t}}\right)$, and $\eta^{\mu \nu}=\operatorname{diag}(1,-1)$. According to Eq. (3), the equation of characteristics becomes

$$
\gamma^{2}\left(\partial_{\tilde{x}} \tilde{\varphi}-\frac{v}{c^{2}} \partial_{\tilde{t}} \tilde{\varphi}\right)^{2}=0,
$$

so that the family of characteristics comes out as the general solution of the ordinary differential equation $\gamma v d \tilde{x}+\gamma c^{2} d \tilde{t}=0$, or equivalently,

$$
u_{\mu} d x^{\mu}=0 .
$$

One finds

$$
\tilde{\varphi}(\tilde{x}, \tilde{t})=\gamma \tilde{t}+\gamma \frac{v}{c^{2}} \tilde{x}=t=\text { const },
$$

with $t$ being the proper time measured in a comoving frame $K$. Recalling Eq. (9), we explicitly see that the characteristics are invariant under Lorentz boosts; cf. Sec. II.

With Eq. (31), the relativistic equivalent to the characteristic Cauchy problem (13) is the following: Find a solution to Eq. (27) which satisfies prescribed values at the initial manifold $\tilde{t}+\left(v / c^{2}\right) \tilde{x}=0$. Clearly, this problem is well-posed only for $\tilde{t}+\left(v / c^{2}\right) \tilde{x}>0$. Its exponential solution can easily be found from Eq. (14) by making use of the Lorentz transformation, $t=\gamma t+\gamma\left(v / c^{2}\right) \tilde{x}$ and $x=\gamma \tilde{x}+\gamma v \tilde{t}$.

Now, the solutions of Eq. (27) that Hiscock and Lindblom [2] examine satisfy periodic initial data $\sim e^{i \tilde{k} \tilde{x}}$ $(\tilde{k} \in \mathbb{R})$ on the non-characteristic line $\tilde{t}=0$. Taking

$$
\vartheta(\tilde{x}, \tilde{t}) \sim e^{i \tilde{k} \tilde{x}+\tilde{\Gamma} \tilde{t}},
$$

they find the dispersion relation

$$
\gamma \alpha \frac{v^{2}}{c^{4}} \tilde{\Gamma}^{2}-\left(1+2 i \gamma \alpha \frac{v}{c^{2}} \tilde{k}\right) \tilde{\Gamma}-\gamma \alpha \tilde{k}^{2}+i v \tilde{k}=0,
$$

see Eq. (67) of Ref. [2]. For $\tilde{k} \neq 0$ the two roots $\tilde{\Gamma}_{1,2}(\tilde{k})$ are complex. The real parts satisfy the conditions

$$
\begin{gathered}
\tilde{\Gamma}_{\mathrm{R} 1}+\tilde{\Gamma}_{\mathrm{R} 2}=\frac{c^{4}}{\gamma \alpha v^{2}}>0, \\
\tilde{\Gamma}_{\mathrm{R} 1} \tilde{\Gamma}_{\mathrm{R} 2}=-\left(\Gamma_{\mathrm{I} 1}-\frac{c^{2} \tilde{k}}{v}\right)^{2} \leq 0,
\end{gathered}
$$

which imply that one of the two modes diverges as $\tilde{t} \rightarrow \infty$. ¿From this the authors of Ref. [2] conclude that the Landau-Lifshitz theory of relativistic hydrodynamics is unstable (in the sense that small spatially bounded departures from equilibrium will diverge with time) and hence must be abandoned as a physically realistic theory.

We disagree with this conclusion. The reason is that the above non-characteristic Cauchy problem is not wellposed, in complete analogy to its non-relativistic equivalent, Eqs. (8), (22). [Note that Eq. (23) equals Eq. (33) if $\beta=v / c$.] Its solution does not depend continuously on the initial data and therefore does not meet one of the three mathematical requirements to be posed with regard to realistic physical problems (cf. Sec. II). So it is the type of the initial-value problem here which is physically unacceptable, and not the instability of the resulting solution.

Nevertheless, it is illuminating to interpret the Hiscock-Lindblom solution in terms of wave propagation (while ignoring the fact that the domain of definition of 
the above Cauchy problem is restricted to the half space $\{(\tilde{x}, \tilde{t}) \mid \tilde{x} \in \mathbb{R}, \tilde{t} \geq 0\})$. The general solution consists of two damped waves traveling to the regions $\tilde{t}>0$ and $\tilde{t}<0$, respectively. Referring again to the discussion of the sideways problem, Sec. III, this is a consequence to be expected. Since the initial manifold crosses the characteristics 31 ), the information (given at $\tilde{t}=0$ ) propagates (along the characteristic lines $\tilde{t}+\left[v / c^{2}\right] \tilde{x}=$ const) in both directions. One thus gets two modes, each transporting the effects of the initial data in the respective region.

It is important to note that the appearance of a mode running backwards in time in non-comoving frames is directly related to the infinite signal speeds accompanying the parabolic equation. In fact, it is well-known 11 that "superluminal acausality" in a Lorentz frame $K$ causes "chronological acausality" in any other Lorentz frames $\tilde{K}$. For example, consider a process in a frame $K$ whereby an event $\mathcal{P}_{1}=(x, t)$ causes another event $\mathcal{P}_{2}=(x+\Delta x, t+\Delta t)$ at superluminal velocity $U>c$. Let the time difference be $\Delta t>0$ so that $\Delta x=U \Delta t>0$. Then, in a frame $\tilde{K}$, moving with velocity $v$ relative to $K$, we have $\Delta \tilde{t}=\gamma \Delta t-\gamma\left(v^{2} / c\right) \Delta x=\gamma \Delta t\left(1-v U / c^{2}\right)$. If $c^{2} / U<v<c$, this yields $\Delta \tilde{t}<0$. This means that in $\tilde{K}$ the signal goes backward in time, or equivalently, that the response $\mathcal{P}_{2}$ precedes the stimulus $\mathcal{P}_{1}$.

Now, as we have seen in Sec. III, the superluminal acausality in $K$ does not lead to any physical consequences; it is automatically precluded by a restriction to the hydrodynamic range of validity. Accordingly, we see from Eqs. (34) and (35) that the damping rate of the mode which propagates in the past is bounded below by $\tilde{\Gamma}_{\mathrm{R}+} \geq c^{4} / \gamma \alpha v^{2}$. With $\tau$ denoting the characteristic microscopic time in $K$ (referred to as the collision time in dilute systems), and $\tilde{\tau}=\gamma \tau$ the respective time in $\tilde{K}$, we thus have

$$
\tilde{\Gamma}_{\mathrm{R}+} \tilde{\tau}=\frac{c^{4} \tau}{\alpha v^{2}} \gg 1 .
$$

(For instance, taking $\alpha$ as the heat conductivity of a piece of iron, one has $\alpha \approx 10^{-5} \mathrm{~m}^{2} / \mathrm{s}$ and $\tau \approx 10^{-12} \mathrm{~s}$, so that $\tilde{\Gamma}_{\mathrm{R}+} \tilde{\tau} \approx 10^{-10}$.) This shows that the mode running backwards in time $\tilde{t}$ decays on a time-scale that is much shorter than the microscopic one, and hence is far outside the hydrodynamic regime.

Let us now come back to the non-characteristic Cauchy problem. Since the domain of definition of the solution is $\{(\tilde{x}, \tilde{t}) \mid \tilde{x} \in \mathbb{R}, \tilde{t} \geq 0\}$, the mode reflecting the chronological acausality is defined only for positive times $\tilde{t}$. As a consequence, the superposed solution does not depend continuously on the initial data, and the problem is illposed. With other words, prescribing Cauchy conditions on a non-characteristic hypersurface in a 2-dimensional flat spacetime does not lead to a realistic physical problem. Such a problem might be obtained only if one prescribes the value of the solution at infinity (cf. the sideways problem in Sec. III), or if the Cauchy data are prescribed on one of the characteristics.
Now, what are the physical conclusions after the mathematical ones have been drawn? Given the fact that the standard Cauchy problem is ill-posed in the LandauLifshitz theory, that one cannot arbitrarily prescribe initial data of physical quantities on a boosted time slice, are we to conclude that the theory is defunct - and to be replaced by one in which the Cauchy problem is wellposed? Is the capability to accommodate arbitrary initial data on boosted time slices a sine qua none for a healthy, physical theory? We believe the answer is no, for two different reasons, although we concede that an affirmative answer may also be upheld. Our reasons are

- All differential equations belonging the the hydrodynamic theory and accounting for coarse-grained, irreversible physics - including the diffusion equation - possess a preferred inertial system, in which the material is at rest. This is not true with respect to microscopic theories for which the Cauchy problem is known to be well-posed in any inertial frame, consider for instance the vacuum Maxwell equations.

- More importantly, in our simple example considered above, we actually know what the initial data on a boosted time slice $\tilde{t}=0$ correspond to in the rest frame, namely to an ill-posed, sideway problem arising from data on a non-characteristic spacelike line $c t-\gamma(v / c) x=0$. This connection is completely general, and especially independent of the Landau-Lifshitz theory. Therefore, an insistence on the well-posedness of Cauchy problems for boosted systems amounts to requiring a new type of differential equation for the rest frame, ones for which the sideway problem is well-posed. Now, there are few equations in physics which are better confirmed than the rest frame diffusion equations as we know them: for temperature, velocity and concentration. And it seems highly unlikely that one can change something as basic as the ill-posedness of the sideway problem without destroying the agreement with the experiments.

Resigning to the fact that the standard Cauchy problem on a boosted time slice may be ill-posed, then clearly, there is no reason whatever to abandon the LandauLifshitz theory.

\section{GENERALIZATION TO CASES OF MORE THAN ONE SPATIAL DIMENSION}

Let us finally examine the general case in which the spatial dimension of the diffusion equation is greater than one. Consider the covariant equation (28), with $\mu$ running now from 1 to 4 . As we have seen in Sec. IV, its characteristics are determined by the ordinary differential equation 


$$
u_{\mu} d x^{\mu}=0
$$

which assigns to each point $x^{\mu}$ an infinitesimal characteristic 3-flat normal to the direction of the time-like velocity $u^{\mu}$ at that point. The crucial point now is that generally these infinitesimal 3-flats do not integrate to 3-surfaces $\varphi\left(x^{\mu}\right)=$ const.

To see this, let us suppose that a general integral $\varphi\left(x^{\mu}\right)=$ const exists. Then, we have

$$
d \varphi=\left(\partial_{\mu} \varphi\right) d x^{\mu}=0,
$$

and hence

$$
\partial_{\mu} \varphi=\lambda u_{\mu}
$$

with some $\lambda=\lambda\left(x^{\mu}\right)$. Since $\partial_{\mu} \partial_{\nu} \varphi=\partial_{\nu} \partial_{\mu} \varphi$, this yields

$$
\partial_{\mu} u_{\nu}-\partial_{\nu} u_{\mu}=\lambda^{-1}\left(u_{\mu} \partial_{\nu} \lambda-u_{\nu} \partial_{\mu} \lambda\right)
$$

from which $\varepsilon^{\mu \nu \alpha \beta} u_{\nu} \partial_{\alpha} u_{\beta}=0$ or

$$
\begin{aligned}
& u_{\alpha}\left(\partial_{\nu} u_{\mu}-\partial_{\mu} u_{\nu}\right) \\
& \quad+u_{\mu}\left(\partial_{\alpha} u_{\nu}-\partial_{\nu} u_{\alpha}\right)+u_{\nu}\left(\partial_{\mu} u_{\alpha}-\partial_{\alpha} u_{\mu}\right)=0
\end{aligned}
$$

results. Equation (41) is a necessary condition for integrability. It can be proved (see eg. 12 that it is also sufficient, ie., if it is satisfied, a general integral exists. [Note that Eq. (41) is identically satisfied for $\alpha, \mu, \nu=1,2$. Thus, in the case of one spatial dimension, characteristics can always be found.] finds

Multiplying the condition of integrability with $u^{\alpha}$, one

$$
\Delta^{\mu \alpha} \Delta^{\nu \beta}\left(\partial_{\alpha} u_{\beta}-\partial_{\beta} u_{\alpha}\right)=0
$$

So, characteristic hypersurfaces exist only if the background equilibrium state is non-rotating. This fact has led various authors to believe that generally the parabolic diffusion equation lacks an initial-value formulation, and hence turns out not to be viable. This, however, would be true only if the non-characteristic Cauchy problem is ill-posed, just like it is in the case of one spatial dimension. Unfortunately, very little seems to be known about the non-characteristic Cauchy problem in spacetimes with more than one spatial dimension. Further work should give clarity here.

* e-mail: liu@itp.uni-hannover.de

[1] L. D. Landau and E. M. Lifshitz, Fluid Mechanics (Pergamon, Oxford, 1987).

[2] W. A. Hiscock and L. Lindblom, Phys. Rev. D 31, 725 (1985).

[3] W. Israel and J. M. Stewart, Ann. Phys. 118, 341 (1979); I. S. Liu, I. Müller, and T. Ruggeri, ibid. 169, 191 (1986); R. Geroch and L. Lindblom, ibid. 207, 394 (1991).
[4] R. Geroch, J. Math. Phys. 36, 4226 (1995).

[5] L. Lindblom, Ann. Phys. 247, 1 (1996).

[6] H. D. Weymann, Amer. J. Phys. 35, 488 (1967).

[7] S. R. de Groot, W. A. van Leeuwen, and Ch. G. van Weert, Relativistic Kinetic Theory (North-Holland, Amsterdam, 1980), Ch. VI $\S 1$ e.

[8] G. B. Nagy, O. E. Ortiz, and O. A. Reula, J. Math. Phys. 35, 4334 (1994). (1987).

[9] S. G. Mikhlin, Mathematical Physics, an Advanced Course (North-Holland, Amsterdam, 1970).

[10] J. Rauch, Partial Differential Equations (SpringerVerlag, New York, 1991).

[11] W. Rindler, Introduction to Special Relativity (Clarendon Press, Oxford, 1991), Sec. 7.

[12] E. L. Ince, Ordinary Differential Equations (Dover Publications, New York, 1956). 